\title{
NICE atrial fibrillation guideline snubs wearable technology: a missed opportunity?
}

\author{
Authors: Andre Briosa e Gala, ${ }^{\mathrm{A}}$ Michael TB Pope, ${ }^{\mathrm{A}}$ Milena Leo, ${ }^{\mathrm{B}}$ Trudie Lobban ${ }^{\mathrm{C}}$ and Timothy R Betts ${ }^{\mathrm{D}}$
}

Atrial fibrillation (AF) is the most common sustained cardiac arrhythmia and a growing public health epidemic. In the UK, over 1.3 million people have a diagnosis of AF and an estimated 400,000 remain undiagnosed. AF-related strokes account for a quarter of all strokes and, as AF episodes are often asymptomatic, are still often the first manifestation of AF. Early diagnosis and initiation of oral anticoagulation, where appropriate, may prevent some of these thromboembolic strokes. Public Health England is committed to decrease the incidence of AF-related strokes and has sponsored initiatives aimed at improving AF detection by promoting the uptake of wearable technologies. However, the National Institute for Health and Care Excellence (NICE) has not recommended wearable technology in their recent AF diagnosis and management guidelines (NG196). Diagnostic accuracy of single-lead electrocardiography (ECG) generated by the latest iteration of wearable devices is excellent and, in many cases, superior to general practitioner interpretation of the 12-lead ECG. High-quality ECG from wearable devices that unequivocally shows $A F$ can expedite AF detection. Otherwise, there is a real risk of delaying AF diagnosis with the potential of devastating consequences for patients and their families.

KEYWORDS: atrial fibrillation, stroke prevention, digital health technology, wearables, NICE

DOI: 10.7861/clinmed.2021-0436

\section{Introduction}

Atrial fibrillation (AF) is the most commonly encountered cardiac arrhythmia. In the UK, over 1.3 million people have an AF diagnosis with a further 400,000 remaining undiagnosed. ${ }^{1}$

Authors: ${ }^{\text {A }}$ cardiology research fellow, John Radcliffe Hospital, Oxford, UK and University of Southampton, Southampton, UK; ${ }^{B}$ cardiology consultant, John Radcliffe Hospital, Oxford, UK;


Association, Stratford upon Avon, UK; ${ }^{D}$ cardiology consultant, John Radcliffe Hospital, Oxford, UK, University of Oxford, Oxford, UK and NIHR Oxford Biomedical Research Centre, Oxford, UK
AF is associated with significant morbidity and mortality. ${ }^{2}$ On average, there are $40 \mathrm{AF}$-related strokes in England each day which are known to be more severe and disabling than non-AF related strokes and, for many, prove fatal. ${ }^{3}$ Public Health England's (PHE's) The NHS Long Term Plan is committed to decreasing the annual stroke rate by improving AF detection and optimising its management across the UK. ${ }^{4}$

Oral anticoagulation (OAC) is a highly effective prevention strategy for AF-related strokes, reducing the stroke risk by twothirds. ${ }^{5}$ However, up to a third of AF patients are asymptomatic and, unfortunately, an ischaemic stroke is still often the first presentation of $A F^{6,7}$ Early diagnosis and initiation of OAC, where appropriate, may prevent some of these thromboembolic strokes. To this effect, PHE together with the Academic Health Science Networks have championed the deployment of innovative digital solutions, including a 6,338 mobile electrocardiography (ECG) device roll-out programme in 2018 aimed at improving AF detection. ${ }^{8}$ It is therefore surprising that the National Institute for Health and Care Excellence (NICE) has not supported this technology in their recent $\mathrm{AF}$ diagnosis and management guidance (NG196). ${ }^{9}$

\section{Digital health revolution}

Technological advancements have led to a plethora of novel noninvasive devices, many commercially available, including patches, smartphones, wearables (watches, bands and rings) and handheld devices, which can detect and monitor arrhythmias and detect possible AF (Fig 1). Initially, volumetric variations in the peripheral microcirculation detected by photoplethysmography (PPG) was used to measure heart rate variability and peak-to-peak changes to detect AF (Fig 2). ${ }^{10}$ PPG signals have several inherent limitations that increase the number of false-positive detections of AF, such as requiring good contact with the skin and, thus, are very susceptible to noise and artefacts from changes in pressure and motion. ${ }^{11}$ The latest generation of wearables incorporate ECG sensor units. These have the advantage of generating high-quality limb-lead ECGs with the patient at rest, discriminating artefact noise from actionable arrhythmias, and are easily exported and reviewed by clinicians, improving diagnostic accuracy. ${ }^{12-14}$

\section{Atrial fibrillation guidelines}

The recently released (April 2021) NICE NG196 AF guidelines failed to incorporate wearable and handheld technology in their diagnostic pathway. Instead, NICE continues to advocate 


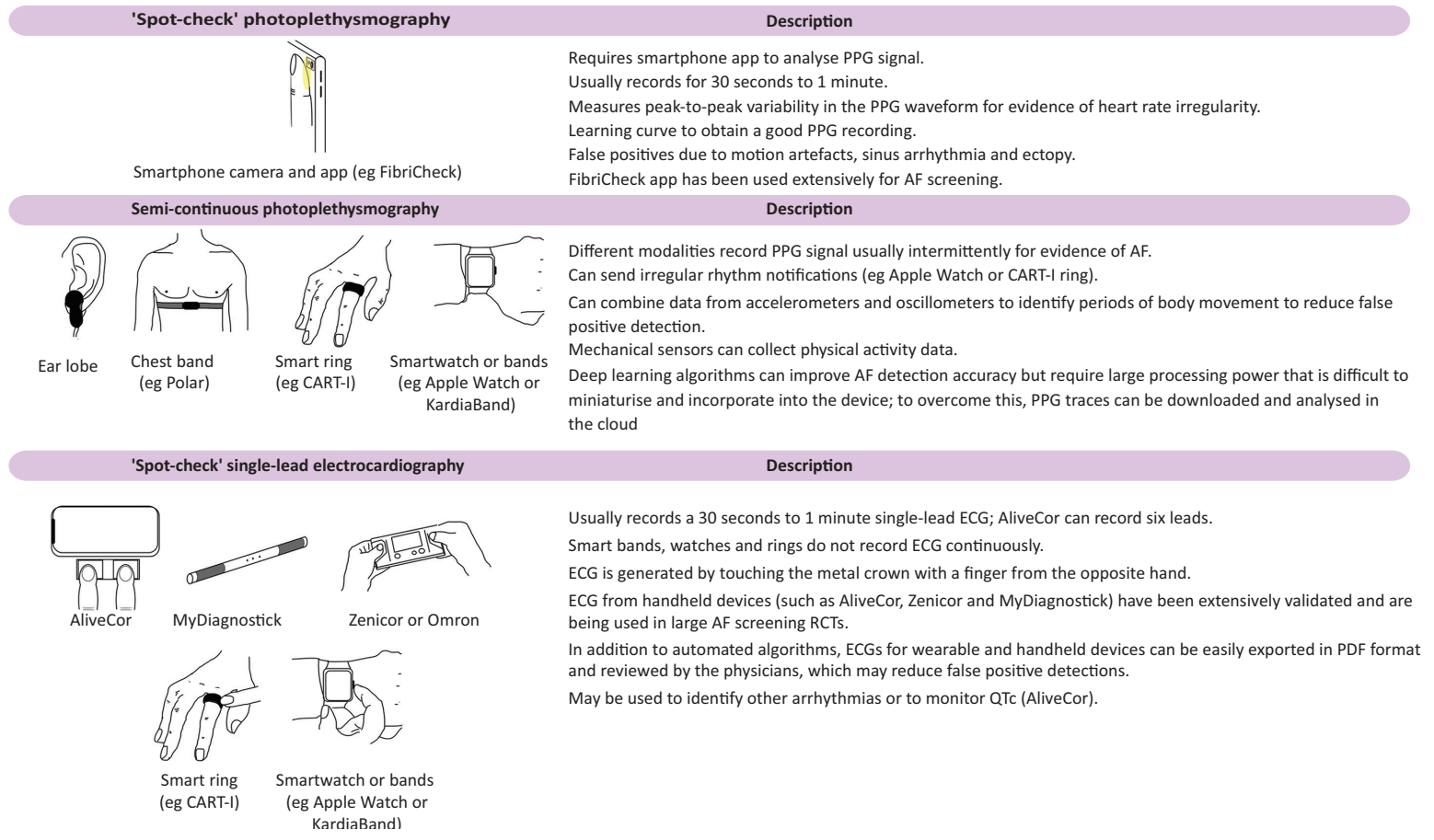

Fig 1. Devices used for atrial fibrillation screening. $A F=$ atrial fibrillation; $P P G=$ photoplethysmography; $R C T s=$ randomised controlled trials.

pulse palpation followed by 12-lead ECG for the detection and diagnosis of persistent AF and longer periods of monitoring (Holter or implantable cardiac monitors) for paroxysmal AF. ${ }^{9}$ This, of course, raises obvious concerns that delays in obtaining a 12-lead ECG in primary care (often not available at the time of a general practitioner (GP) appointment) or limited access to ambulatory monitoring will translate into fewer AF detections, leading to increased number of AF-related strokes while people await a diagnosis and are not anticoagulated. This guidance also brings about uncertainty in clinical practice: should a physician ignore single-lead ECGs from wearables devices that clearly show AF from a symptomatic patient; should OAC be withheld until subsequent monitoring confirms AF; and how should we counsel these patients and their families if they suffer an AF-related stroke while awaiting further investigations.

In stark contrast, the European Society of Cardiology (ESC) took a different stance by accepting as evidence of clinical AF an irregular rhythm without discernible P-waves on a 30 -second single-lead ECG generated by a validated wearable device and interpreted by a physician. ${ }^{6}$ It, however, rightly advocates caution, highlighting that there is still uncertainty in this fast-moving field and calls for high-quality, large, randomised studies. This pragmatic approach relies upon physicians exercising their clinical judgement when appraising ECG waveforms from wearable devices and deciding if it represents a 'true' AF episode, a falsepositive detection or if further investigations are required.

To establish the diagnostic accuracy of wearable devices, NICE reviewed studies that compared either automatic detection and/ or clinician interpretation of both PPG and ECG waveforms to a 12lead ECG interpreted by an expert clinician, usually a cardiologist, as the gold-standard. Although NICE acknowledged that newer devices are accurate and show promise, it did not make any recommendations as it felt a definitive test needs 'almost perfect' sensitivity and specificity. ${ }^{9}$ However, if we exclude studies that rely on automatic detection or PPG signals, and only examine those with physician interpretation of a single-lead ECG (as endorsed by the ESC), the sensitivity and specificity is high (ranging from 0.84 to 0.97 and 0.86 to 0.97 , respectively; Table 1$)^{12-24}$ In some cases, it was even superior to the diagnostic accuracy of GP interpretation of a 12-lead ECG (sensitivity of 0.79 and specificity of 0.92) as observed in the SAFE study, which included 2,595 primary care patients (Fig 3). ${ }^{25}$ Interestingly, in their current review, there was a discrepancy in the qualitative assessment of studies also included in the NICE DG35 (lead-I ECG devices for detection of symptomatic AF in primary care), having been considered previously as 'low' risk of bias but were now upgraded to 'serious' or 'very serious' risk of bias. ${ }^{12,16,17,21,22}$ The rationale for this is unclear.

The AF Association, a UK-based charity that promotes AF awareness by educating patients and healthcare professionals, has released an 'AF white paper' endorsing clinically validated ECG-based wearable devices and expressed concerns that the new NICE recommendations will delay AF detection. ${ }^{26}$

\section{Wearable devices and AF screening}

The use of wearable technology may extend the opportunity for detection to significant sections of the population with the potential for much to gain. This was illustrated in the Apple Heart Study that was conducted virtually and recruited 419,297 participants in only 8 months. ${ }^{27}$ The main aim was to determine AF detection accuracy of the Apple Watch; it was not designed to investigate outcomes. It included mainly a young healthy population and, unsurprisingly, only $0.5 \%$ had an irregular rhythm 
a



b

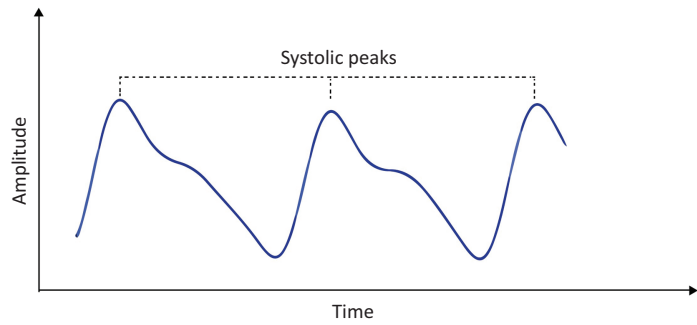

C Atrial fibrillation (irregular peak-to-peak)

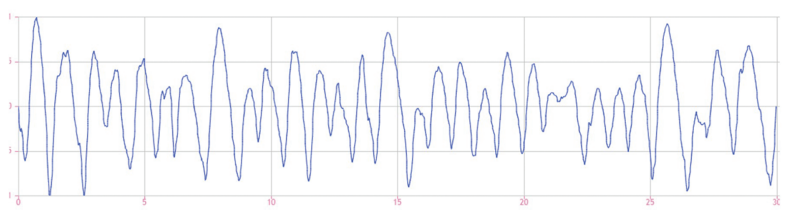

Sinus rhythm (regular peak-to-peak)

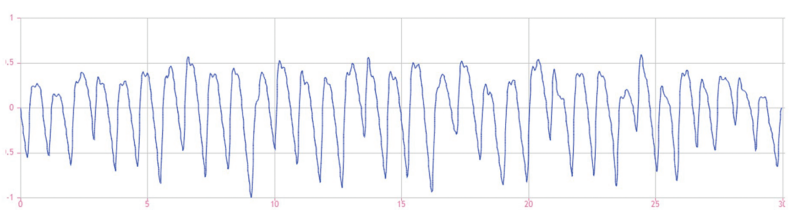

Artefacts and low amplitude PPG



Fig 2. a) Photoplethysmography (PPG) relies on reflected light to measure blood volume changes in the microvasculature. b) A PPG wave form has a systolic and diastolic component and changes in both amplitude and period can identify heart rate irregularity. c) Examples of a 30-second PPG waveform recorded by the CART-I Ring (SkyLabs). PPG $=$ photoplethysmography.

notification. The positive predictive value was $84.9 \%$ despite low AF prevalence.

Until recently, there were limited randomised clinically relevant outcome data to support systematic screening of AF. Wearable devices may provide a more convenient low-cost tool for widespread screening. The STROKESTOP study presented at Annual congress of the European Heart Rhythm Association 2021 assessed systematic screening in patients aged 75-76 years old with handheld single-ECG device (Zenicor) twice daily for 2 weeks versus routine care. ${ }^{28}$ The intention-to-treat analysis showed only a modest $4 \%$ reduction $(p=0.045)$ in the primary outcomes (composite of thromboembolic events, severe bleeding and death) at minimum follow-up of 5.6 years. An important caveat is that of those offered screening with a wearable device, only slightly more than half actively participated. In this group, initiation of OAC led to a $24 \%$ reduction in ischaemic stroke. The SAFER (ISRCTN72104369) and HEARTLINE (NCT04276441) trials will recruit hundreds of thousands of primary care patients $\geq 65$ years old and provide further evidence of the feasibility and efficacy of population screening with wearable technology.

\section{Challenges and unanswered questions}

'Self-initiated' rhythm monitoring with wearable technology is likely to identify short infrequent asymptomatic AF episodes in apparently healthy individuals that are of unclear clinical significance. It is important to bear in mind that net clinical benefit of thromboprophylaxis with OAC has only been demonstrated in patients with AF detected by a 12-lead ECG and a significant number of patients with consumer devices represents a healthier/younger cohort with a likely different stroke risk. Evidence from longitudinal studies of device-detected atrial high-rate episodes (also referred to as subclinical AF) suggest a significant yearly stroke risk (2.8/100 per person-years). This is numerically smaller than patients with clinical AF and the role of OAC is not yet established. ${ }^{29}$ The AF burden and episode duration that merits anticoagulation is also unknown. In studies of patients with device-detected AF, the threshold for increased thromboembolic risk was met following AF episodes lasting longer than 5 minutes (MOST), 1 hour (SOS), 5.5 hours (TRENDS) and 24 hours (Botto et al, Cappuci et al and post-hoc analysis of ASSERT). ${ }^{30-35}$ Two randomised controlled trials (ARTESiA (NCT01938248) and NOAH AFNET 6 (NCT02618577)) are currently recruiting and may provide further insights regarding stroke prevention in subclinical AF. The ESC recommends considering OAC in patients with high stroke risk and subclinical AF episodes longer than 24 hours.

There are also valid apprehensions that commercially available devices may not be validated to the same standard as medicalgrade devices and hence generate a large number of false-positive detections leading to harm from overtreatment and unwarranted downstream investigations. Keeping abreast with the diagnostic accuracy of wearables devices with different algorithms and criteria for notification of irregular rhythms, frequent software updates and patches will also prove challenging.

Acceptance of wearable devices within the cardiology community continues to steadily increase. A survey by Maninger et al of over 500 cardiologists showed that $83 \%$ would diagnose AF based on a single-lead wearable device ECG and $72 \%$ would start oral anticoagulation. ${ }^{36}$ We are already experiencing an enthusiastic uptake of wearable devices from arrhythmia patients. Indeed, in TeleCheck-AF, rhythm assessment is performed by the FibriCheck app 1-week prior teleconsultation obviating the need for an ECG in primary care. ${ }^{37}$ This international project was set up in response to the COVID-19 pandemic and has already recruited over 4,000 patients in 41 centres.

Without acknowledgment of their clinical utility, it will be challenging to procure the necessary funding and support from all stakeholders, including regulatory bodies, for a comprehensive evaluation of current clinical services with a view of updating current infrastructure, data management systems, security and governance to facilitate the seamless integration of wearable data into electronic patient records. 


\begin{tabular}{|c|c|c|c|c|}
\hline Index test & Study & $\mathrm{n}$ & $\begin{array}{l}\text { Sensitivity } \\
(95 \% \mathrm{CI})\end{array}$ & $\begin{array}{l}\text { Specificity } \\
(95 \% \mathrm{CI})\end{array}$ \\
\hline \multirow[t]{6}{*}{ AliveCor (lead-I ECG) } & Desteghe, $2017^{12}$ & 445 & $1.00(0.83-1.00)$ & $0.98(0.95-0.99)$ \\
\hline & Haberman, $2015^{17}$ & 130 & $0.94(0.73-1.00)$ & $0.99(0.95-1.00)$ \\
\hline & Williams, $2015^{13}$ & 99 & $0.93(0.77-0.99)$ & $0.76(0.64-0.85)$ \\
\hline & Koltowski, $2019^{20}$ & 100 & 0.928 & 1.00 \\
\hline & Himmelreich, $2019^{18}$ & 219 & $1.00(0.85-1.00)$ & $1(0.98-1.00)$ \\
\hline & & $\begin{array}{l}\text { Total: } \\
993\end{array}$ & $\begin{array}{l}\text { Pooled: } 0.95 \\
(0.88-0.99)\end{array}$ & $\begin{array}{l}\text { Pooled: } 0.96 \\
(0.81-0.99)\end{array}$ \\
\hline KardiaBand (equivalent to lead-I ECG) & Bumgamer, $2018^{14}$ & 100 & $0.88(0.79-0.94)$ & $0.86(0.76-0.93)$ \\
\hline Merlin ECG event recorder (single-lead ECG) & Kearley, $2014^{19}$ & 1,000 & 0.939 & 0.901 \\
\hline \multirow[t]{3}{*}{ Omron HeartScan HCG (single-lead ECG) } & Kearley, $2014^{19}$ & 1,000 & 0.944 & 0946 \\
\hline & Kaleschke, $2019^{22}$ & 568 & $0.99(0.96-1.00)$ & $0.96(0.94-0.98)$ \\
\hline & & $\begin{array}{l}\text { Total: } \\
1,568\end{array}$ & $\begin{array}{l}\text { Pooled: } 0.97 \\
(0.93-1.00)\end{array}$ & $\begin{array}{l}\text { Pooled: } 0.95 \\
(0.93-0.97)\end{array}$ \\
\hline MyDiagnostick (single-lead ECG; one measure over 60 seconds) & Desteghe, $2017^{12}$ & 445 & $0.85(0.62-0.97)$ & $0.95(0.92-0.98)$ \\
\hline Zenicor (bipolar lead I; one measure of 10 seconds) & Doliwa, $2009^{16}$ & 100 & $0.96(0.86-1.00)$ & $0.92(0.81-0.98)$ \\
\hline Beurer ME90 (single-lead ECG; one measure of 30 seconds) & Brito, $2018^{23}$ & 126 & $0.84(0.60-0.97)$ & $1.00(0.97-1.00)$ \\
\hline ECG-Bone (single-lead ECG; unclear duration) & Proesman, $2019^{24}$ & 223 & $0.90(0.83-0.95)$ & $0.97(0.92-0.99)$ \\
\hline
\end{tabular}

CI = confidence interval; ECG = electrocardiography.

\section{Conclusion}

AF is a growing public health epidemic with an estimated 400,000 patients remaining undiagnosed in the UK. AF-related strokes account for a quarter of all strokes and oral anticoagulation reduces the stroke risk by two-thirds. Undoubtedly, there are many challenges and hurdles prior to widespread adoption of digital health technologies and rigorous studies are warranted. However,
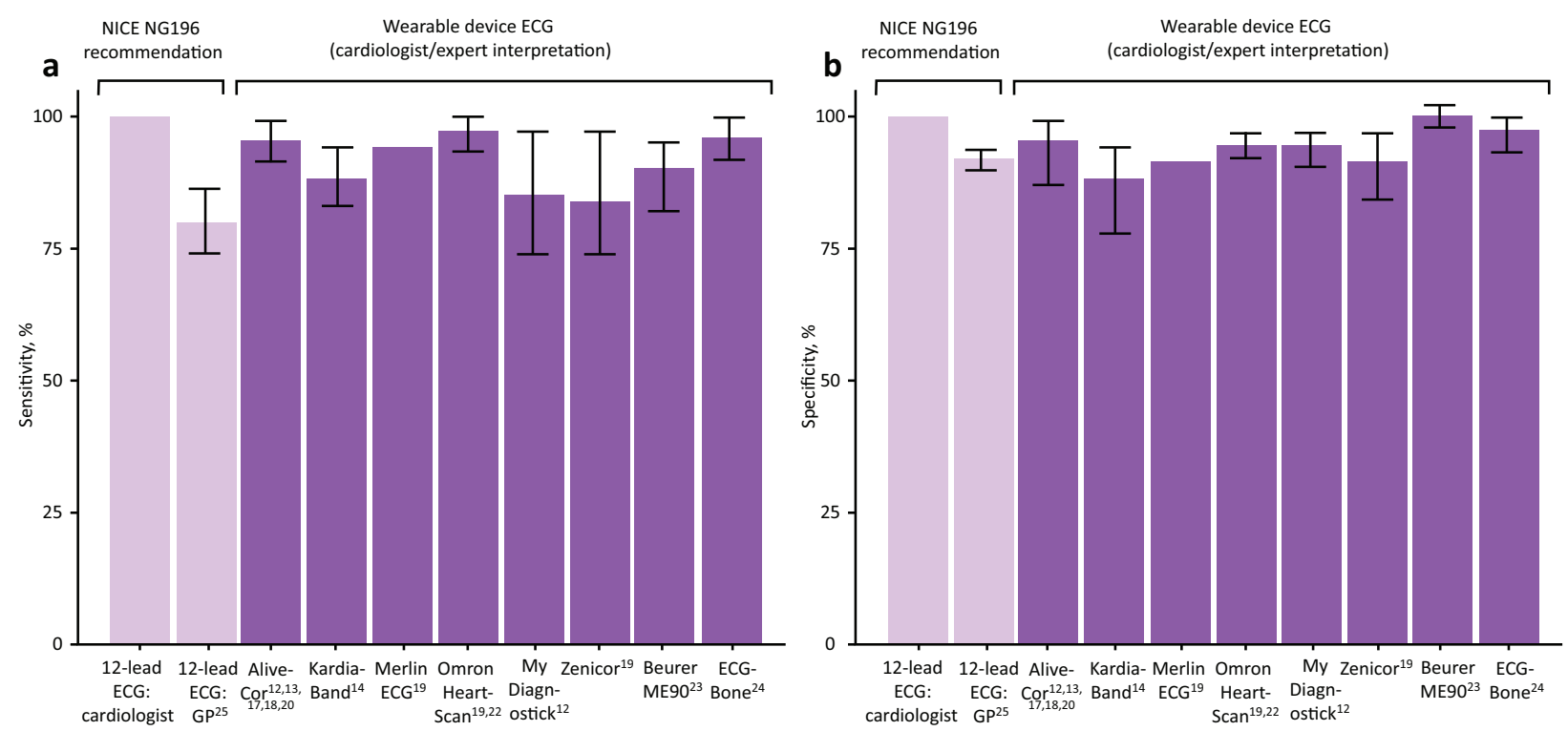

Fig 3. Sensitivity and specificity of 12-lead electrocardiography (ECG) interpreted by cardiologist and general practitioners (GPs; endorsed by National Institute for Health and Care Excellence (NICE) as gold standard) and single-lead ECG from wearable devices interpreted by an expert physician included in NICE Expert Review B (NG196). ${ }^{9}$ 
rather than not integrating any wearable technology, a more nuanced approach is desirable; incorporating high-quality singlelead ECG data from the latest generation of wearable devices that unequivocally show AF may expedite AF detection. Otherwise, there is a real risk of delaying AF diagnosis with the potential for devastating consequences for patients and their families.

\section{Funding}

Timothy R Betts and Andre Briosa e Gala received a Heart Research UK novel and emerging technologies grant to investigate the role of wearable devices in $\mathrm{AF}$ management (RG2689/20/22).

\section{References}

1 Public Health England. Public Health Profiles. Public Health England. https://fingertips.phe.org.uk/search/atrial

2 Odutayo A, Wong CX, Hsiao AJ et al. Atrial fibrillation and risks of cardiovascular disease, renal disease, and death: systematic review and meta-analysis. BMJ 2016;354:14482.

3 Atrial fibrillation prevalence estimates for local populations. https:// www.gov.uk/government/publications/atrial-fibrillation-prevalenceestimates-for-local-populations [Last accessed 12 May 2015].

4 NHS England. The NHS Long Term Plan. NHS, 2019. www.england. nhs.uk/long-term-plan [Accessed 11 May 2021].

5 Hart RG, Pearce LA, Aguilar MI. Meta-analysis: antithrombotic therapy to prevent stroke in patients who have nonvalvular atrial fibrillation. Ann Intern Med 2007;146:857-67.

6 Hindricks G, Potpara T, Dagres N et al. 2020 ESC Guidelines for the diagnosis and management of atrial fibrillation developed in collaboration with the European Association for Cardio-Thoracic Surgery (EACTS): The Task Force for the diagnosis and management of atrial fibrillation of the European Society of Cardiology (ESC) Developed with the special contribution of the European Heart Rhythm Association (EHRA) of the ESC. Eur Heart ] 2021:42:373-498.

7 Dilaveris PE, Kennedy HL. Silent atrial fibrillation: epidemiology, diagnosis, and clinical impact. Clinical Cardiology 2017;40:413-8.

8 The AHSN Network. Independent evaluation of the AHSN Network mobile ECG roll-out programme. The AHSN Network, 2019.

9 National Institute for Health and Care Excellence. Atrial fibrillation: diagnosis and management: NICE guideline [NG196]. NICE, 2021. www.nice.org.uk/guidance/ng196 [Accessed 13 May 2021].

10 Kamal AA, Harness JB, Mearns AJ. Skin photoplethysmography-a review. Comput Methods Programs Biomed 1989;28:257-69.

11 Li KHC, White FA, Tipoe T et al. The current state of mobile phone apps for monitoring heart rate, heart rate variability, and atrial fibrillation: narrative review. JMIR Mhealth Uhealth 2019;7:e11606.

12 Desteghe L, Raymaekers Z, Lutin M et al. Performance of handheld electrocardiogram devices to detect atrial fibrillation in a cardiology and geriatric ward setting. Europace 2017;19:29-39.

13 Williams ], Pearce K, Benett I. The effectiveness of a mobile ECG device in identifying AF: sensitivity, specificity and predictive value. $\mathrm{Br}$ ] Cardiol 2015;22:70-2.

14 Bumgarner JM, Lambert CT, Hussein AA et al. Smartwatch algorithm for automated detection of atrial fibrillation. J Am Coll Cardiol 2018;71:2381-8.

15 Caillol T, Strik M, Ramirez FD et al. Accuracy of a smartwatchderived ECG for diagnosing bradyarrhythmias, tachyarrhythmias, and cardiac ischemia. Circ Arrhythm Electrophysiol 2021;14:e009260

16 Doliwa PS, Frykman V, Rosenqvist M. Short-term ECG for out of hospital detection of silent atrial fibrillation episodes. Scand Cardiovasc ] 2009;43:163-8
17 Haberman ZC, Jahn RT, Bose R et al. Wireless smartphone ECG enables large-scale screening in diverse populations. J Cardiovasc Electrophysiol 2015;26:520-6.

18 Himmelreich JCL, Karregat EPM, Lucassen WAM et al. Diagnostic Accuracy of a Smartphone-Operated, Single-Lead Electrocardiography Device for Detection of Rhythm and Conduction Abnormalities in Primary Care. Ann Fam Med 2019;17:403-11.

19 Kearley K, Selwood M, Van Den Bruel A et al. Triage tests for identifying atrial fibrillation in primary care: a diagnostic accuracy study comparing single-lead ECG and modified BP monitors. BMJ Open 2014:4:e004565.

20 Koltowski L, Balsam P, Glowczynska R et al. Kardia Mobile applicability in clinical practice: A comparison of Kardia Mobile and standard 12-lead electrocardiogram records in 100 consecutive patients of a tertiary cardiovascular care center. Cardiol ] 2021:28:543-8.

21 Vaes B, Stalpaert S, Tavernier K et al. The diagnostic accuracy of the MyDiagnostick to detect atrial fibrillation in primary care. BMC Fam Pract 2014;15:113.

22 Kaleschke G, Hoffmann B, Drewitz I et al. Prospective, multicentre validation of a simple, patient-operated electrocardiographic system for the detection of arrhythmias and electrocardiographic changes. EP Europace 2009;11:1362-8.

23 Brito R, Mondouagne LP, Stettler C, Combescure C, Burri H. Automatic atrial fibrillation and flutter detection by a handheld ECG recorder, and utility of sequential finger and precordial recordings. J Electrocardiol 2018;51:1135-40.

24 Proesmans T, Mortelmans C, Van Haelst R et al. Mobile phonebased use of the photoplethysmography technique to detect atrial fibrillation in primary care: diagnostic accuracy study of the FibriCheck App. JMIR mHealth uHealth 2019;7:e12284.

25 Mant J, Fitzmaurice DA, Hobbs FDR et al. Accuracy of diagnosing atrial fibrillation on electrocardiogram by primary care practitioners and interpretative diagnostic software: analysis of data from screening for atrial fibrillation in the elderly (SAFE) trial. BMJ 2007;335:380.

26 AF Association. AF White Paper: Put People First. AF Association, 2021. www.heartrhythmalliance.org/afa/uk/af-white-paper-putpeople-first

27 Perez MV, Mahaffey KW, Hedlin $\mathrm{H}$ et al. Large-scale assessment of a smartwatch to identify atrial fibrillation. N Engl ] Med 2019;381:1909-17.

28 Svennberg E. Late Breaking Clinical Trials: Benefits of systematic screening for atrial fibrillation - the STROKESTOP-study. EHRA 2021 Online Congress, 2021. https://digital-congress.escardio.org/EHRACongress/sessions/511-late-breaking-clinical-trials

29 Mahajan R, Perera T, Elliott AD et al. Subclinical device-detected atrial fibrillation and stroke risk: a systematic review and metaanalysis. Eur Heart ] 2018;39:1407-15.

30 Glotzer TV, Hellkamp AS, Zimmerman ] et al. Atrial high rate episodes detected by pacemaker diagnostics predict death and stroke: report of the Atrial Diagnostics Ancillary Study of the MOde Selection Trial (MOST). Circulation 2003;107:1614-9.

31 Boriani G, Glotzer TV, Santini M et al. Device-detected atrial fibrillation and risk for stroke: an analysis of $>10000$ patients from the SOS AF project (Stroke preventiOn Strategies based on Atrial Fibrillation information from implanted devices). Eur Heart ] 2013;35:508-16.

32 Glotzer TV, Daoud EG, Wyse DG et al. The relationship between daily atrial tachyarrhythmia burden from implantable device diagnostics and stroke risk: the TRENDS study. Circ Arrhythm Electrophysiol 2009;2:474-80

33 Botto GL, Padeletti L, Santini M et al. Presence and duration of atrial fibrillation detected by continuous monitoring: crucial implications for the risk of thromboembolic events. J Cardiovasc Electrophysiol 2009;20:241-8. 
34 Capucci A, Santini M, Padeletti L et al. Monitored atrial fibrillation duration predicts arterial embolic events in patients suffering from bradycardia and atrial fibrillation implanted with antitachycardia pacemakers. J Am Coll Cardiol 2005;46:1913-20.

35 Van Gelder IC, Healey JS, Crijns HJGM et al. Duration of devicedetected subclinical atrial fibrillation and occurrence of stroke in ASSERT. Eur Heart ] 2017;38:1339-44.

36 Manninger M, Zweiker D, Svennberg E et al. Current perspectives on wearable rhythm recordings for clinical decision-making: the wEHRAbles 2 survey. EP Europace 2021;23:1106-13.
37 Linz D, Pluymaekers NAHA, Hendriks JM. TeleCheck-AF for COVID19: A European mHealth project to facilitate atrial fibrillation management through teleconsultation during COVID19. Eur Heart ] 2020;41:1954-5.

Address for correspondence: Dr Andre Briosa e Gala, John Radcliffe Hospital, Oxford University Hospitals NHS Foundation Trust, Headley Way, Oxford OX3 7BA, UK. Email: andre.gala@ouh.nhs.uk Twitter: @briosa_gala; @popemtb 